Revue scientifique sur la conception et l'aménagement de l'espace

\title{
Que nous dit la végétation de la cohérence spatiale, biologique et paysagère de la " ceinture verte » de la région Île-de-France?
}

What information does vegetation provide about the spatial and biological coherence of the green belt landscape in the Ile-de-France region?

\section{Fabien Roussel}

\section{OpenEdition}

Journals

Édition électronique

URL : http://journals.openedition.org/paysage/9843

DOI : $10.4000 /$ paysage. 9843

ISSN : 1969-6124

Éditeur :

École nationale supérieure du paysage de Versailles-Marseille, Institut national des sciences appliquées Centre Val de Loire - École de la nature et du paysage, École nationale supérieure d'architecture et de paysage de Bordeaux, École nationale supérieure d'architecture et de paysage de Lille, Agrocampus Angers

\section{Référence électronique}

Fabien Roussel, «Que nous dit la végétation de la cohérence spatiale, biologique et paysagère de la " ceinture verte » de la région île-de-France? », Projets de paysage [En ligne], 13 | 2015, mis en ligne le 31 décembre 2015, consulté le 04 septembre 2020. URL : http://journals.openedition.org/paysage/ 9843 ; DOI : https://doi.org/10.4000/paysage.9843

Ce document a été généré automatiquement le 4 septembre 2020

Projets de paysage 


\title{
Que nous dit la végétation de la cohérence spatiale, biologique et paysagère de la « ceinture verte » de la région Île-de-France?
}

\author{
What information does vegetation provide about the spatial and biological \\ coherence of the green belt landscape in the Ile-de-France region?
}

Fabien Roussel

1 En dépit de l'engouement pour l'esthétique champêtre magnifiée par les peintres - en Île-de-France, l'école de Barbizon et les impressionnistes -, c'est sans référence ni à la couleur verte ni à ses nuances que les premiers projets de ceintures végétalisées autour des villes ont d'abord été envisagés au tournant des $\mathrm{XIX}^{\mathrm{e}}$ et $\mathrm{Xx}^{\mathrm{e}}$ siècles : comme des espaces libres d'urbanisation, où alternaient les «petits coins vert épinard" de l'Albertine de Proust (1924) et les parcs boisés. Un siècle plus tard, l'ambition des aménageurs pour les couronnes périurbaines et rurales dépasse le seul fait de contenir l'étalement urbain. On repart à la recherche de cette esthétique champêtre tout en l'enrichissant d'objectifs écologiques, économiques ou sociaux : maintien du patrimoine biologique et paysager, préservation des espaces et des ressources agricoles, accueil de loisirs verts, cadre de vie de nature, etc. (Poulot, 2013). Il s'agit de remettre en paysage et en nature les ceintures périurbaines et d'en tirer des services pour les habitants des villes comme pour les visiteurs.

2 La proposition d'une ceinture verte pour l'agglomération parisienne au début des années 1980 par le conseil régional ${ }^{1}$ a reposé sur des ambiguïtés. D’un côté, dans la logique de cet outil d'aménagement conçu et mis en œuvre depuis la fin du xix siècle pour limiter l'étalement des agglomérations urbaines ( $c f$. infra), il s'agit de contribuer à l'effort de planification urbaine porté par l'État depuis le schéma directeur de 1965. D'un autre côté, à la différence par exemple de la Green Belt londonienne, le positionnement géographique même de la ceinture francilienne, à cheval sur 
l'agglomération et la couronne rurale, entre 10 et 30 kilomètres du centre de Paris, ne rend guère possible la réalisation de l'objectif de limitation de l'étalement urbain. Dès le départ, elle se présente donc comme une mosaïque d'espaces plus ou moins morcelés, plus ou moins urbains, forestiers ou agricoles. Ces limites floues comme l'absence de cadre réglementaire strict, son échec à contenir l'étalement urbain (Barbieri, 2002), de même que le recours à d'autres principes organisateurs (trames vertes, fronts urbains) l'ont depuis reléguée dans l'anonymat au sein des institutions régionales.

3 À l'heure du redéploiement de la métropole parisienne, notamment au travers des projets multiformes qui accompagnent la mise en place du Grand Paris, et de la montée en puissance des problématiques environnementales générées par la ville, il est temps de réexaminer le projet de ceinture verte pour l'agglomération parisienne en revenant sur le terrain, sur les espaces non bâtis à la limite de deux modes majeurs d'occupation du sol, urbains d'un côté, ruraux de l'autre. Malgré la discrétion du projet francilien, le principe même de préservation et de mise en valeur de ces espaces face à l'urbanisation est toujours d'actualité. Il s'agit bien de préserver une altérité écologique et paysagère à la ville.

Dans cet article, nous prenons le chemin de la végétation pour discuter le sens et la valeur de la couleur verte de la ceinture, couleur qui agrège toutes les nuances de cette altérité. Ce chemin nous mène vers les espaces végétaux semi-naturels et vers le paysage que leur agencement génère et que des projets encadrent. La recherche concernant les espaces non-bâtis situés sur les pourtours de l'agglomération parisienne n'est pas nouvelle. Elle a jusqu'à présent exploré la dimension agricole, selon une approche épistémologique (Poulot, 2009), ou bien au regard des projets de développement régionaux (Brédif et Pupin, 2012), ou encore sous l'angle d'enjeux patrimoniaux (Darly, 2010). Ces recherches ne répondent pas cependant à la question de la cohérence verte de ces espaces, c'est-à-dire de leur structuration écologique et paysagère. Nous inscrivons cette question de la cohérence dans le cadre de réflexions classiques en géographie sur les notions de continuité et de discontinuité spatiales, qui invitent aussi à se saisir des notions d'homogénéité ou d'hétérogénéité spatiales (Alexandre et Génin, 2008). Ces interrogations guident notre analyse spatiale de la végétation dans les franges urbaines. Y répondre est nécessaire pour clarifier l'échelle et la portée des projets de paysage, au premier rang desquels celui de ceinture verte.

5 Nous revenons tout d'abord sur l'évolution de la signification de la couleur verte des ceintures périurbaines dans l'histoire de l'aménagement, pour ainsi mieux situer le projet qui a vu le jour en Île-de-France. Nous détaillons ensuite le chemin conceptuel, méthodologique et spatial qui nous mène de l'échelle régionale à l'échelle parcellaire. Nous fondons notre approche sur l'étude des espaces végétaux semi-naturels et sur une méthode d'emboîtement des échelles du paysage qui nous conduit à proposer une typologie des grands types de franges. Enfin, nous nous appuyons sur l'un des secteurs mis en évidence dans la typologie, la plaine de Pierrelaye et ses environs, la forêt de Montmorency et la vallée de l'Oise, pour illustrer l'hétérogénéité spatiale et biologique dans laquelle sont menés des projets locaux de mise en valeur et discuter ainsi de leur rôle dans le contexte régional de ceinture verte. 


\section{De la green belt à la ceinture verte, retour sur les étapes d'une transposition}

\section{De quoi le vert des ceintures périurbaines est-il le nom ?}

6 La couleur verte accolée aux ceintures périurbaines des grandes métropoles européennes n'a pas toujours eu la connotation environnementale qu'on lui connaît aujourd'hui. Quand cette "forme d'aménagement urbain d'échelle régionale» (Alexandre, 2013) est imaginée par les urbanistes à la fin du $\mathrm{XIX}^{\mathrm{e}}$ siècle, faisant le constat de l'urbanisation galopante sous l'impulsion de la révolution industrielle, c'est avec l'objectif de lutter contre l'étalement urbain et de maintenir des espaces libres. Cette ambition constitue l'ADN des ceintures vertes à travers le monde occidental au cours du Xx ${ }^{e}$ siècle (Amati, 2008).

7 Elle se teinte d'abord d'hygiénisme social, en particulier à Paris qui aurait pu être pionnier en la matière, à l'emplacement de l'ancienne ligne de fortification construite par Thiers en 1840, et de sa zone non aedificandi (Alexandre et Génin, 2014). La zone est alors occupée par un ensemble d'habitats précaires dans lequel il s'agit de mettre de l'ordre. Mais l'idée de ceinture verte est abandonnée au profit d'aménagements hybrides mêlant parcs, espaces sportifs et infrastructures publiques dont l'héritage est toujours bien visible aujourd'hui le long des boulevards des Maréchaux.

C'est finalement à Berlin et à Londres que le projet est mis en œuvre. Le plan Jansen à Berlin est adopté en 1929 et pose une double ceinture d'espaces libres d'urbanisation (Alexandre, 2013). Il faut attendre 1944 et le plan Abercrombie pour voir le Grand Londres s'appuyer ouvertement sur le principe de green belt. Le vert y prend alors une coloration esthétique et patrimoniale. L'aversion des urbanistes britanniques à l'égard des paysages hybrides des franges urbaines est patente dès 1909 dans les propos de Raymond Unwin : " that irregular fringe of half-developed suburb, and half-spoiled country which forms such a hideous and depressing girdle around modern growing towns " (cité par Amati, 2008). Le concept de green belt a aussi été retenu en Grande-Bretagne par des groupes en faveur de la préservation des paysages dans une logique esthétique et normative. Londres constitue le cas de ceinture verte le plus abouti à ce jour malgré certains assouplissements et l'émergence de problématiques liées au coût du foncier et à l'éloignement du logement (Gant, Robinson, et Fazal, 2011).

Le concept a essaimé à travers le monde occidental sous influence britannique. 14 villes britanniques en sont progressivement «équipées ». Puis, au cours des années 1960 et 1970, le Canada, la Nouvelle-Zélande, l'Australie voient émerger une ceinture verte autour de leurs grandes agglomérations. Cette notoriété du concept s'exporte jusqu'à Hong Kong, au Japon et en Corée du Sud (Amati, 2008).

10 L'essor des ceintures vertes à travers le monde occidental a été une occasion pour l'aménagement en tant que discipline d'accéder à la modernité. Les aménageurs britanniques ont vu dans les ceintures vertes un outil rationnel et universel d'aménagement des agglomérations dans un esprit scientifique et moderniste: " Overall, therefore, while the concept was deliberately spread by British planners keen to use the green belt as a "poster boy" for their nascent dsicipline, it was also copied wholesale by some cities. » (Ibid.) Le succès ou l'échec des tentatives a pu ainsi s'expliquer par le caractère trop normatif des projets et le manque de prise en compte des contextes locaux. 
11 Il faut attendre la fin du $\mathrm{xx}^{\mathrm{e}}$ siècle pour voir les problématiques écologiques donner à la couleur verte des ceintures périurbaines une envergure nouvelle. Les nouveaux enjeux de gouvernance locale, les problématiques écologiques générées par les villes, le développement de nouvelles manières de penser la nature en ville (les trames vertes en France par exemple) sont pour Marco Amati l'occasion d'interroger sous un nouveau jour les ceintures vertes, dans son ouvrage de référence sur le sujet Urban Green Belts in the Twenty-first Century (2008).

12 Notre étude de la ceinture verte de la région île-de-France s'inscrit, elle aussi, dans cette réflexion, mais souhaite mettre l'accent sur le rôle que la végétation joue dans l'émergence ou non de franges urbaines « vertes ».

\section{Le projet perdu de ceinture verte francilienne}

Le concept de ceinture verte apporte une réponse stricte à la question des limites entre l'espace urbain et l'espace rural, selon une vision discontinue de l'espace (Alexandre et Génin, 2008). Nous venons de le voir, c'est comme tel qu'il a été pensé pour le Grand Londres de l'après-guerre. La région Île-de-France en a décidé autrement en posant la ceinture verte exactement à cheval sur le cœur d'agglomération et la couronne rurale, davantage dans une logique de continuité spatiale. Un critère de proportion en espaces ouverts (c'est-à-dire non bâtis, au sens large) par commune compris entre 40 et $60 \%$ a été retenu par les institutions : en deçà, l'urbain dense, au-delà, la couronne rurale, domaine des parcs naturels régionaux. Il s'agit bien de répondre au besoin de protection des espaces non bâtis dans cet entre-deux. Aujourd'hui, près de 4 millions de personnes vivent dans la ceinture verte. La dernière proposition en date pour son périmètre s'appuie sur un découpage communal réalisé selon le critère d'espaces ouverts arrêté (cf. supra) ${ }^{2}$. 
Figure 1. Emprise spatiale de la ceinture verte de la région île-de-France à l'échelle de l'agglomération parisienne

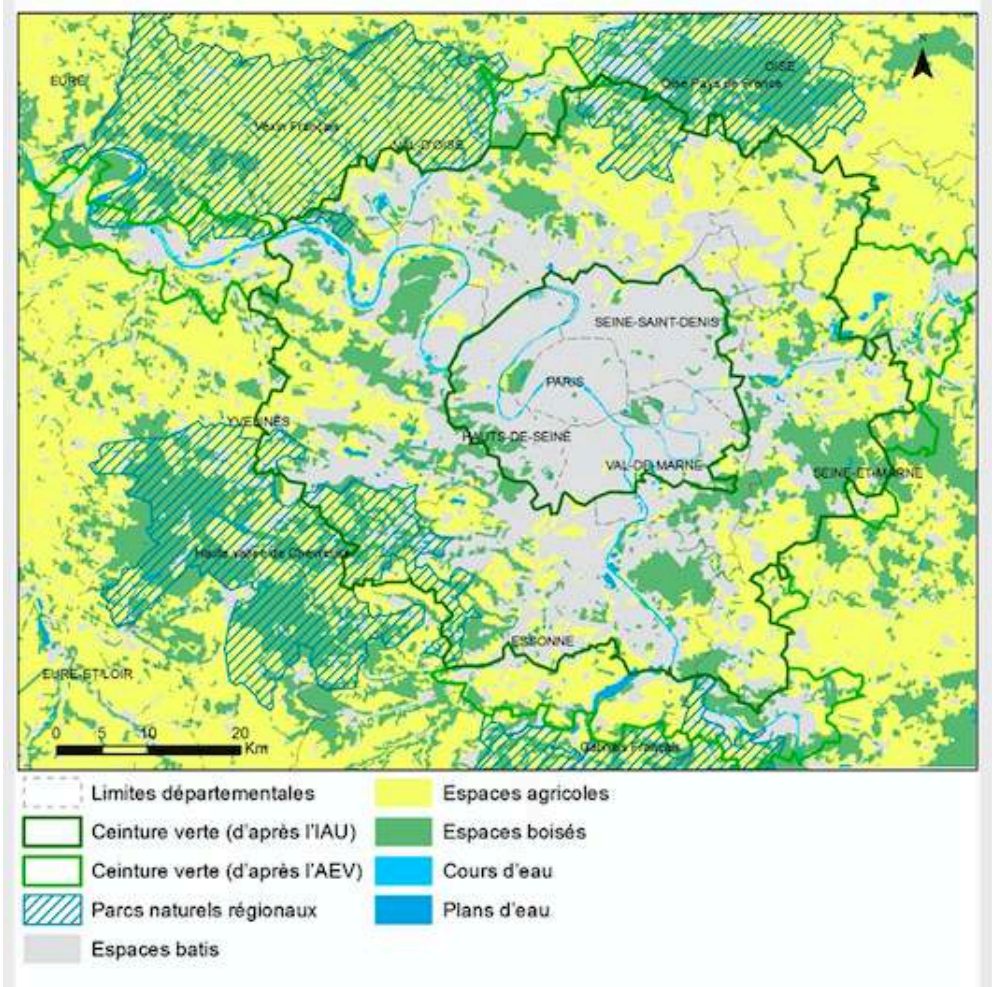

Source : Fabien Roussel, 2015, d'après la carte IGN TOP 100 Tourisme et découverte $n^{\circ} 190$ Paris, Chantilly, Fontainebleau de juillet 2011 et l'Institut d'aménagement et d'urbanisme de la région île-deFrance.

De la délibération régionale de 1983, en passant par l'apogée qu'a constituée le plan vert régional de 1995, pour finir avec le schéma directeur de la région Île-de-France (SDRIF) de 2013, la ceinture verte francilienne a été prise dans le jeu des compétences institutionnelles et des décisions politiques. Elle a en effet été l'occasion pour la région de s'appuyer, face à l'État alors en charge du SDRIF, sur un concept d'aménagement fort (Amati, 2008) pour mettre en œuvre sa compétence en matière d'espaces verts. D'après les personnes interrogées au sein des institutions régionales, le jeu de l'alternance politique semble ensuite avoir scellé le sort du plan vert de 1995 et, dans une moindre mesure, de la ceinture verte qui en constituait l'élément central. La ceinture verte est dès lors reléguée au second plan des SDRIF de 1994 et de 2013 et reste depuis dissimulée au sein de l'Institut d'aménagement et d'urbanisme (IAU), d'une part, et de l'Agence des espaces verts (AEV), d'autre part. Il est cependant curieux de relever que l'AEV a pris l'initiative d'étendre le périmètre de la ceinture verte aux grandes vallées de la Seine et de la Marne, notamment sans concertation aucune avec ses partenaires régionaux (voir figure ci-dessus). Aujourd'hui, le projet est donc éparpillé au sein du SDRIF sans réel fil conducteur, et grâce à la seule persévérance, pas toujours coordonnée, des instituts régionaux en charge de la question. Notons enfin que la ceinture verte n'est pas prise en compte dans les projets du Grand Paris.

Dès 2002, l'IAU pose un constat mitigé au moment de faire un bilan de la ceinture verte et relève la contradiction des développements urbains proposés par le SDRIF de 1994 dans le périmètre de la ceinture verte (Barbieri, 2002). L'Institut poursuit la réflexion pendant une dizaine d'années, s'interrogeant sur la « réinvention » d'un espace de vie 
(Laruelle et Legenne, 2005) pour finalement en revenir à son «invention» (Laruelle et Legenne, 2012). De la logique d'outil d'aménagement pour lutter contre l'étalement urbain, les institutions ont glissé vers la notion de projet de territoire : la ceinture verte est vue comme un cadre de vie où populations et projets s'appuient sur la valorisation environnementale des espaces ouverts comme voie de reconnaissance d'une moyenne couronne. La nature transitoire de l'espace entre la ville et la campagne est ainsi pleinement assumée, dans une logique continue de l'espace géographique.

En raison de ce parti pris et faute d'un ancrage réglementaire solide, le rôle de frein à l'urbanisation de la ceinture verte s'est trouvé amoindri et la notion même a perdu sa lisibilité spatiale. D'autres façons de concevoir le contrôle de l'étalement urbain ont été retenues au sein des institutions, en particulier la notion de front urbain (IAU Île-deFrance, 2010). Depuis le Grenelle de l'environnement en 2008, les questions de nature en ville ont quant à elle largement été pensées sous l'angle des trames vertes et bleues (Clergeau et Blanc, 2013). Dans les deux cas, ce sont d'autres échelles qui sont considérées. Ces dernières se prêtent mieux à des projets locaux mais ne répondent pas directement à l'échelle régionale, à l'inverse de la ceinture verte.

La logique retenue par les institutions d'un pourcentage d'espaces ouverts par commune ne peut être suffisante pour rendre compte des transitions et/ou des ruptures spatiales à l'œuvre dans les franges externes de l'agglomération parisienne. Il est proposé dans la partie suivante de revenir au terrain et de s'intéresser à l'organisation spatiale de la végétation et aux paysages qu'elle génère.

\section{De la ceinture verte aux espaces végétaux : un emboîtement paysager}

\section{La place de la végétation dans la terminologie des espaces des franges urbaines dans le contexte de ceinture verte}

Dans le but de redonner de la clarté aux enjeux écologiques et paysagers, la couleur verte de la ceinture est approchée sous l'angle de la végétation. Nous concentrons l'analyse sur les espaces végétaux semi-naturels, là où la végétation s'exprime le plus spontanément. Cette analyse porte sur les arrangements spatiaux de ces espaces et sur leur rôle dans les franges de l'agglomération à différentes échelles, sur leur composition botanique et sur les paysages qu'ils génèrent. Les espaces verts urbains (parcs, jardins, pelouses urbaines, etc.) n'ont pas retenu notre attention. Ils sont totalement pris en charge par la ville et intégrés à elle (Alexandre, 2013; Novarina, 2003) malgré des développements récents en faveur d'une gestion différenciée qui laisse plus de place aux dynamiques spontanées (Lizet, 2010).

19 Nous laissons également de côté la notion d'espace ouvert employée par les professionnels de l'aménagement. Elle signifie bien, quant à elle, une altérité aux espaces bâtis. En ce sens elle fait l'objet de recherches en géographie (Banzo, 2015). Son caractère synthétique et malléable a pu être mis en valeur (Poulot, 2013). Cependant, du point de vue biogéographique, ce caractère est problématique : la notion agrège des contextes spatiaux différents sur le plan des structures et dynamiques végétales (massifs forestiers plus ou moins exploités pour la sylviculture, boisements isolés, espaces de cultures plus ou moins intensifs, délaissés végétaux, etc.). De la logique 
initiale des espaces libres d'urbanisation telle qu'elle était invoquée au commencement des ceintures vertes européennes (Alexandre et Génin, 2014), à celle des espaces ouverts aujourd'hui convoquée, c'est toujours une manière de voir par l'urbain: l'altérité est réduite à un espace des possibles. Nous proposons donc d'en revenir à un objet d'étude plus précis et plus neutre du point de vue végétal.

Le caractère semi-naturel des espaces végétaux étudiés indique une intervention humaine avérée sur la végétation qui conserve cependant une structure et des peuplements vivants qui «diffèrent peu de leur état naturel » (Da Lage et Métaillé, 2005). La spontanéité de la flore est donc une clé de lecture majeure mais doit être nuancée : les forêts domaniales sont ainsi peuplées d'individus dont la présence est favorisée par l'activité sylvicole. De même, certaines espèces rencontrées dans les espaces végétaux sont issues de populations plantées dans les cultures, parcs ou jardins. À ce titre, les sciences naturelles les qualifient de "subspontanées ", voire de «naturalisées » quand l'implantation est durable ${ }^{3}$. Ces précautions sémantiques pour qualifier les espaces végétaux des franges urbaines sont nécessaires au moment d'étudier leur hétérogénéité spatiale, biologique et paysagère (voir la dernière partie sur le cas du nord-ouest de l'agglomération parisienne autour de la plaine de Pierrelaye).

21 Passer de l'échelle régionale des franges dans leur ensemble à l'échelle parcellaire qui est celle des espaces végétaux semi-naturels nécessite de proposer une grille de lecture opérante de l'emboîtement des échelles. Le paysage est ici au cœur de la démarche.

\section{L'emboîtement des échelles du paysage}

Pour passer de la région à la parcelle, nous faisons la proposition méthodologique d'un emboîtement des échelles du paysage tel qu'il a été avancé par Michel Godron (Godron et Joly, 2008, Godron, 2012). À l'origine de l'essor de l'écologie des paysages dans les années 1980 avec Richard T. T. Forman (Forman et Godron, 1986), Michel Godron a enrichi les propositions initiales sur les éléments paysagers d'une réflexion proprement géographique sur l'emboîtement des paysages. S'inspirant de la systématique appliquée aux règnes animal et végétal, il pose des types de paysages, puis des familles, des ordres, enfin des classes. Chaque échelle renvoie à des facteurs organisateurs spécifiques agissant eux-mêmes sur des durées particulières :

- les classes de paysages sont gouvernées par les climats zonaux, à l'échelle du 1/10000 $000^{\mathrm{e}}$ (exemple en France : méditerranéen, atlantique, continental) ;

- les ordres sont gouvernés par le climat régional et la géomorphologie, à l'échelle du $1 / 1000000^{\mathrm{e}}$;

- les familles de paysages, de la dizaine à la centaine de kilomètres, sont gouvernées par le climat local et la géomorphologie, mais aussi par l'action de l'homme. Elles comportent des éléments analogues dans des proportions qui peuvent varier;

- les types de paysages, du kilomètre à la dizaine de kilomètres, réunissent des éléments similaires dans des proportions voisines; le rôle de l'homme y est fort, capable de compenser les conditionnements précédents. La pédogenèse et la topographie continuent d'avoir un rôle important ;

- le paysage lui-même ou l'unité paysagère, de l'hectomètre au kilomètre, où les facteurs humains prennent encore davantage de place ; 
- les éléments du paysage enfin, du décamètre à l'hectomètre, où seuls les facteurs humains sont réellement significatifs. l'échelle de l'agglomération parisienne et interroger les jeux des continuités/ discontinuités et de l'homogénéité/hétérogénéité spatiale. La ville, l'openfield ou les massifs forestiers sont des types de paysage au sens de Godron : s'y exprime, à l'échelle du $1 / 100000^{e}$, le rôle prépondérant des êtres humains sans que les conditions topographiques et pédologiques ne cessent d'avoir un impact. En conséquence, le périurbain, en ce qu'il agrège ou juxtapose ces types-là, relève lui aussi de cette échelle. Cet emboîtement nous mène ensuite sans heurt scalaire vers les espaces végétaux, puis vers la flore.

\section{Types de paysage des franges à l'échelle régionale}

Partant d'une approche matérielle du paysage énoncée plus haut, nous avons procédé à la cartographie des grands types d'occupation du sol à l'échelle régionale en nous appuyant sur la carte au $1 / 100000^{\mathrm{e}}$ de l'IGN ${ }^{4}$ : espaces bâtis, espaces agricoles, espaces boisés, espaces en eau. C'est aussi une manière d'appréhender les espaces végétaux dans leurs arrangements généraux en s'appuyant sur les espaces boisés et les espaces agricoles comme une forme certes grossière mais synthétique de ce type d'espace. La carte IGN au $1 / 100000^{e}$ permet en outre d'avoir une vue d'ensemble de l'espace régional. Reprenant la proposition de Michel Godron sur les types de paysages, nous avons appliqué une maille coulissante de $10 \mathrm{~km}$ sur 10 sur la limite de la tache d'agglomération centrale. 
Figure 2. Occupation du sol dans l'espace de la ceinture verte de la région île-de-France

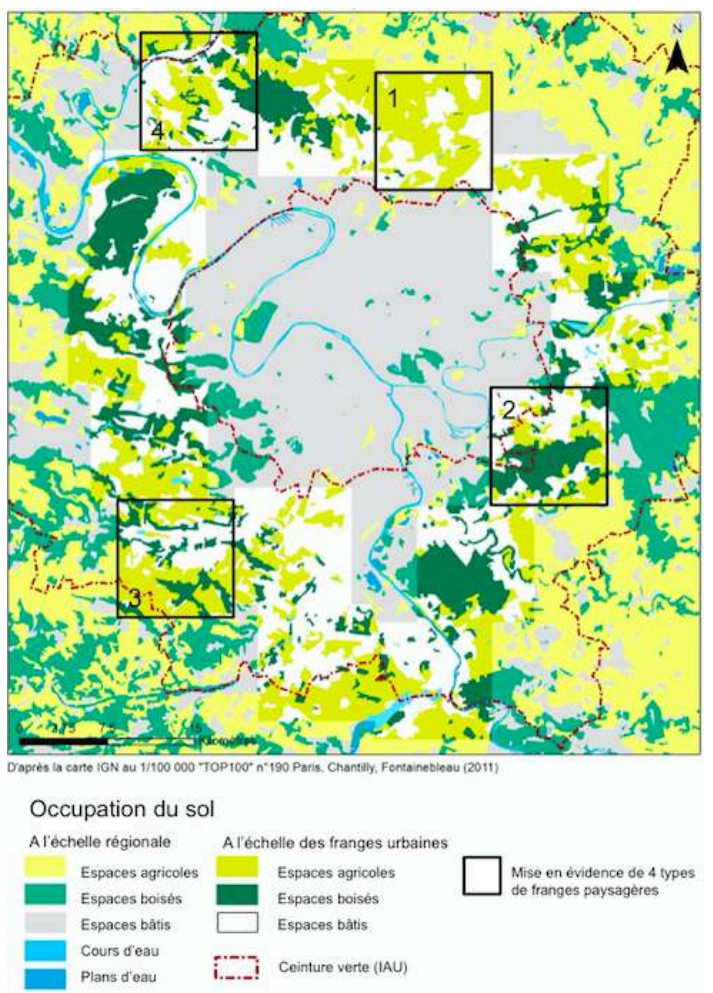

Source : Fabien Roussel, 2015, d'après la carte IGN au 1/100 000 TOP 100 nº 190 Paris, Chantilly, Fontainebleau, 2011.

Nous en avons extrait des «types de paysages » de franges urbaines au sens de Michel Godron. Cette typologie s'appuie sur les arrangements spatiaux des modes d'occupation des sols et met en évidence des contextes spatiaux bien connus.

Le premier type met frontalement en présence l'espace agricole et la ville. Cela ne signifie pas que l'urbanisation soit interrompue. Elle reprend au contraire selon un phénomène de mitage et se propage aussi à partir de ces centres isolés, ce qui a été commenté par ailleurs (Grison, 2012). La dimension forestière est absente, la topographie ne présente pas d'obstacle au développement urbain. Le secteur de Goussainville en est un bon exemple (encadré 1 de la figure ci-dessus). Les projets à venir autour du plateau de Saclay (via le Grand Paris) ou à l'emplacement du Triangle de Gonesse (avec le projet Europa City) illustrent la vulnérabilité de ces espaces de grande culture. Leur contenu écologique peu reconnu, la dimension mondialisée et lointaine de la ressource agricole, le poids exclusif et intensif de celle-ci sur le paysage ne leur permettent pas d'accéder à un statut qui les valoriserait aux yeux des citadins. Ce sont des espaces peu reconnus et peu fréquentés auxquels l'agriculture périurbaine tente d'ouvrir de nouvelles perspectives (Poulot, 2012).

Les massifs forestiers en position de tampon sont une seconde caractéristique des franges de l'agglomération. Leur statut institutionnel (ce sont souvent des forêts domaniales) et patrimonial leur permet de se maintenir sur ces pourtours et de jouer un rôle de frein à l'urbanisation assez efficace. Ce rôle est visible dans le cas de la forêt de Montmorency ou de Notre-Dame (encadré 2 de la figure ci-dessus), c'est moins le cas pour les massifs de Sénart ou de Meudon, progressivement absorbés par l'agglomération et prenant la 
forme d'îles boisées à l'échelle de l'agglomération. Ces grands massifs forestiers produisent des types de paysages fréquentés par les populations et reconnus par elles.

Les secteurs de vallées sont des axes privilégiés et anciens de l'urbanisation parisienne. Le bâti occupe souvent le fond de vallée, en connexion directe avec le cœur d'agglomération, laissant les versants à une couverture forestière et le plateau à l'activité agricole ; le bâti déborde parfois sur les plateaux. La vallée de Chevreuse est un bon exemple de ce cas de figure (encadré 3 de la figure ci-dessus) mais c'est aussi le cas de la vallée de l'Oise et de la Seine en particulier en aval. Dans un style moins marqué, les vallées de l'Yerres, de l'Essonne ou de l'Orge ont également ce profil.

31 Enfin, il existe une quatrième catégorie, zones hétérogènes, où l'agencement des éléments paysagers présente l'aspect d'une mosaïque complexe. Poches agricoles, éclatement des boisements, urbanisation discontinue sont les caractéristiques du secteur de la plaine de Pierrelaye (encadré 4 de la figure ci-dessus) ou de la forêt de Bondy.

Ces types de paysages des limites nous permettent de mettre en évidence des contextes particuliers de franges et donc de nuancer une première fois l'espace de la ceinture verte, et, en outre, de procéder à un échantillonnage pour changer d'échelle et partir observer les paysages eux-mêmes à l'échelle locale.

Pour déterminer les paysages, nous retenons l'échelle du kilomètre, en respectant l'emboîtement scalaire retenu, ce qui correspond à ce qu'un observateur perçoit d'un seul regard. Nous nous appuyons ensuite sur les concepts de l'écologie du paysage, en particulier ceux d'éléments du paysage et de matrice paysagère. La notion de matrice renvoie au fond du paysage, à ce qui en constitue la structure et les dynamiques fondamentales. Trois critères permettent de la mettre en évidence (Forman et Godron, 1986) : la surface relative, le degré de connectivité, le degré de contrôle sur les dynamiques paysagères.

Le secteur de la plaine de Pierrelaye qui inclut aussi une partie de la forêt de Montmorency et de la vallée de l'Oise est ici retenu comme étude de cas.

\section{Hétérogénéité paysagère et projets de paysage : le cas du nord-ouest de l'agglomération (plaine de Pierrelaye- forêt de Montmorency-vallée de l'Oise)}

La plaine de Pierrelaye et ses alentours, la forêt de Montmorency et la vallée de l'Oise sont représentatifs du type 4 «franges urbaines hétérogènes ». Le lien qui unit emprise spatiale des espaces végétaux, composition botanique, arrangements paysagers ainsi que produits et projets de paysage fondés sur la végétation s'y exprime pleinement.

\section{Le poids des matrices paysagères sur l'hétérogénéité du paysage}

Il n'est pas surprenant d'observer trois types de matrice, forestière, agricole, urbaine, dans ce secteur marqué par l'hétérogénéité de l'occupation du sol (soit les mêmes critères qui nous ont servi pour la cartographie au 1/100 000 $)$. Reprenant l'idée d'un 
degré de contrôle de la matrice, nous avançons l'hypothèse que les matrices sont ici en compétition, toutes n'ayant pas le même pouvoir de domination :

- la matrice urbaine est agressive, car portée par des logiques démographiques et économiques puissantes ;

- la matrice forestière est quant à elle plus statique mais elle peut s'appuyer sur des statuts de gestion efficaces et reconnus, tels que les forêts domaniales ;

- la matrice agricole est sans doute la plus vulnérable car sans statut de protection vraiment reconnu, mis à part son classement dans les documents d'urbanisme, et les périmètres régionaux d'intervention foncière (PRIF) portés par l'Agence des espaces verts de la région ou le soutien des parcs naturels régionaux.

Premier constat, chacune ne fait pas preuve de la même tolérance à l'égard des espaces végétaux : la ville dense leur laisse peu de place, la ville lâche leur en laisse davantage. Il en va de même pour l'agriculture: plus l'intensité sera élevée, moins les espaces semi-naturels seront nombreux. Le principe de tolérance est directement lié à l'intensité des activités humaines mais aussi à la topographie et au substrat géologique. Ce sont ces critères qui vont apporter de la nuance au sein des matrices. Mais c'est aussi la proximité avec la matrice urbaine, la plus dominante, qui va jouer.

Par ce biais, nous souhaitons mettre en évidence les dynamiques et les processus spatiaux à l'origine du maintien de la végétation spontanée et subspontanée des espaces urbains, forestiers et agricoles. Il en découle une mosaïque spatiale qui produit une hétérogénéité paysagère.

\section{La végétation : une clé de lecture de la qualité paysagère et des types de projets de paysage}

Végétation et paysage sont intrinsèquement liés via la notion de formation végétale (Alexandre et Genin, 2011) qui fait écho aux caractères physionomiques de la végétation. Ces derniers influent sur le paysage et sont, dans le contexte périurbain, le résultat des interventions humaines selon les modes de gestion, de mise en valeur ou au contraire de délaissés.

Les espaces végétaux constituent des éléments du paysage au sein des matrices paysagères. Ils renvoient à des types de formation végétale : prairies, bois, parcelles forestières, haies, bords de chemin, etc., dans lesquels nous avons mené des relevés botaniques (101 au total sur le secteur étudié). Près de 340 espèces, correspondant à tous les types biologiques, y ont été identifiées.

Derrière cette somme, se profile en réalité une flore typique des milieux tempérés très anthropisés. Les espèces les plus rencontrées en témoignent par leur caractère rudéral et leur plasticité écologique. C'est le cas de la ronce bleuâtre (Rubus caesius) et de la ronce commune (Rubus fruticosus), de l'ortie (Urtica dioica), du lierre (Hedera helix), de l'érable sycomore (Acer pseudoplatanus) et de l'aubépine (Crataegus monogyna). Un tiers des espèces relevées a en outre un caractère nitrophile, confirmant la proximité avec les activités humaines. Un quart est dit ubiquiste, capable de coloniser des milieux divers. 


\section{Des projets locaux de mise en valeur en ordre dispersé}

42 l'éparpillement des espaces végétaux, de leur intrication, de leur hybridation aussi sur le plan biologique. Il en découle une variété de modes de mise en valeur ou, à l'inverse, de mise à l'écart, pour lesquels s'expriment des usages et des fonctions variés qui ont une influence sur les caractéristiques végétales du secteur et, in fine, sur la couleur verte de l'ensemble de la ceinture.

Les espaces végétaux sont parfois pleinement intégrés à un projet patrimonial et paysager local. C'est le cas des berges de l'Oise à Auvers-sur-Oise par exemple.

Figure 3. Les bords de l'Oise à Auvers-sur-Oise

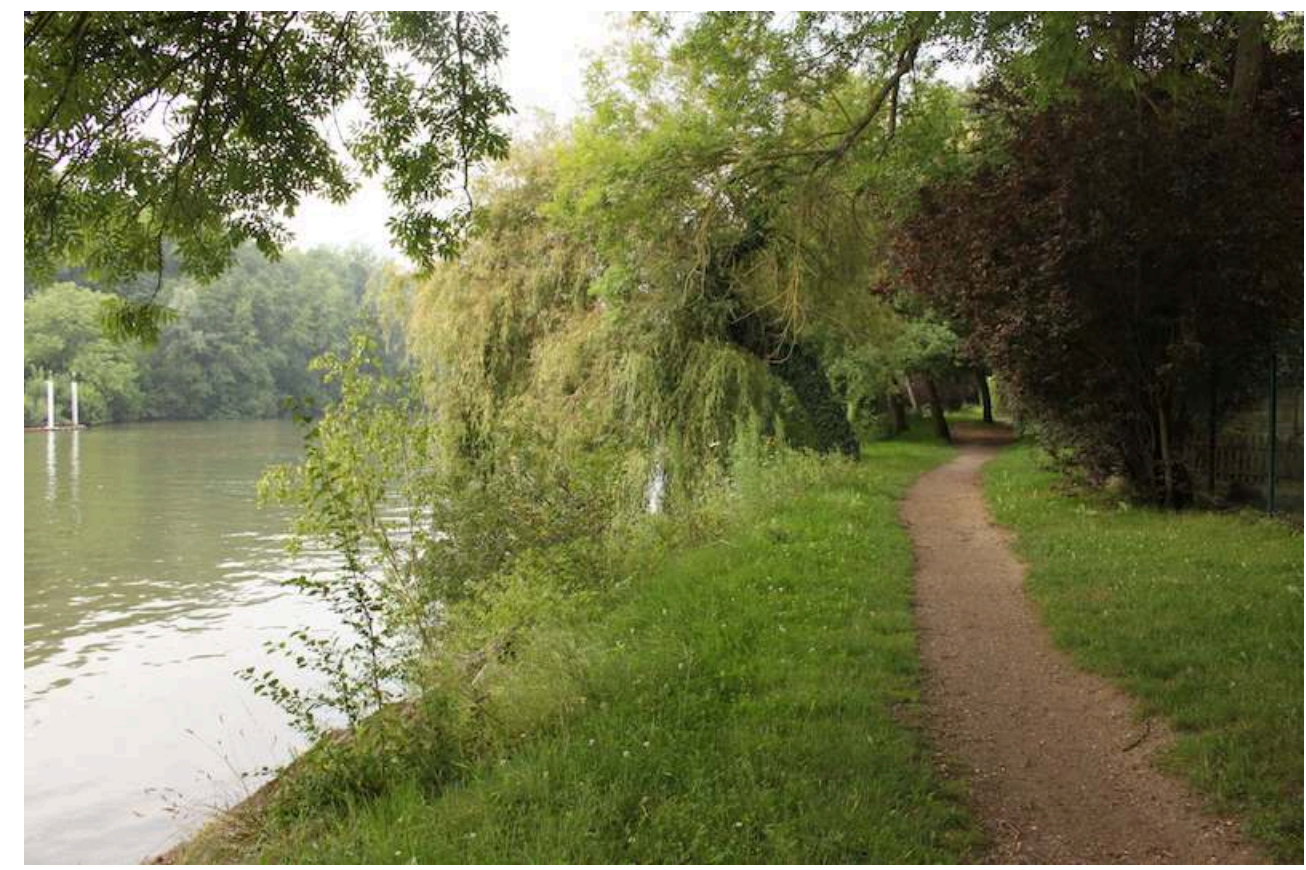

Source : Fabien Roussel, juin 2014.

La flore des bords de l'Oise mêle ainsi des espèces caractéristiques des ripisylves: aulnes (Alnus glutinosa), saules blancs (Salix alba), saules cassants (Salix fragilis), accompagnées d'espèces herbacées hygrophiles telles que la salicaire (Lytrum salicaria), l'épilobe hérissé (Epilobium hirsutum) ou l'eupatoire chanvrine (Eupatorium canabinum) et des espèces anthropophiles telles que l'érable sycomore (Acer pseudoplatanus), la benoite commune (Geum urbanum), le pissenlit (Taraxacum officinal) la grande chélidoine (Chelidonium majus) ou l'ivraie vivace (Lolium perenne). Le paysage qui en résulte traduit cette hybridation.

Les espaces végétalisés sont d'autres fois totalement délaissés, comme en plaine de Pierrelaye, frange marginalisée au sens spatial et même social du terme. Les espaces végétalisés et leur résultante paysagère sont ici hors de tout projet, laissés à la guise des initiatives individuelles propres aux situations de marges: dépôts sauvages, campements spontanés, etc. 
Figure 4. Bois rudéralisés au nord de la plaine de Pierrelaye, sur la commune de Bessancourt

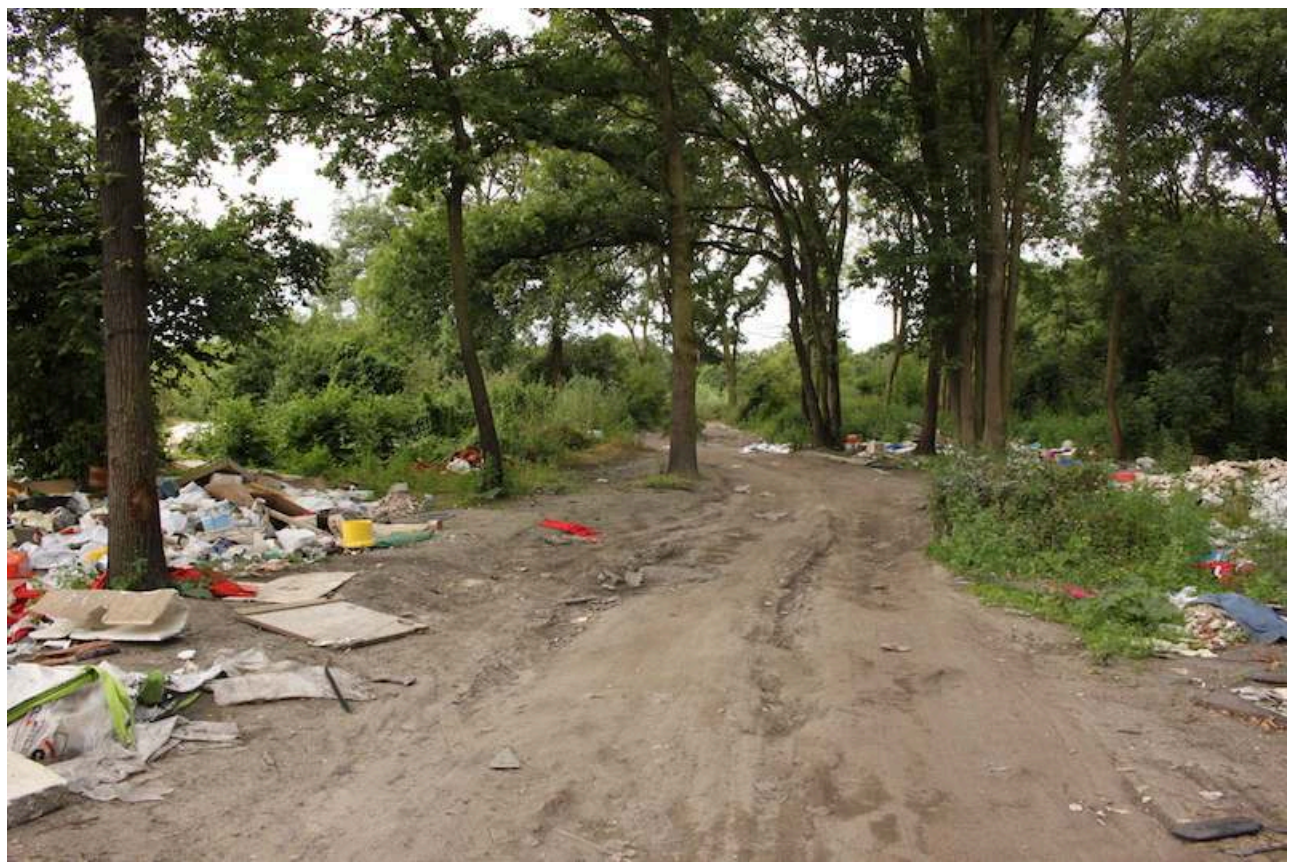

Source : Fabien Roussel, juin 2014.

La flore y est rudérale, caractéristique des milieux perturbés. La structure interne des formations arborées est dense, les strates buissonnantes et arbustives prenant une large place. C'est le lieu de prédilection des aubépines (Crataegus monogyna), des érables sycomores (Acer pseudoplatanus), des ronces bleuâtres (Rubus caesius) et des sureaux noirs (Sambucus nigra). Les lisières, au contact des zones agricoles, voient proliférer orties (Urtica dioica), gaillets gratterons (Galium aparine) ou mûriers (Rubus fruticosus). Les formations herbacées sont conquises par des espèces au caractère fortement social, telles que le cirse des champs (Cirsium arvense) ou l'onagre bisannuelle (Oenothera biennis) qui rendent incertaine l'appréciation paysagère. Le projet d'une forêt pour cet espace n'est pas anodin: il dénote le désarroi des acteurs face à un espace peu appropriable, sur fond d'héritage industriel (pollution des sols). Le projet consiste alors à repartir de zéro pour fabriquer un paysage ex nihilo avec force incantation environnementale.

En situation d'îlot dans la ville, la plaine de Boissy, au nord-ouest de l'agglomération, sur les communes du Plessis-Bouchard et de Beauchamp, réunit tous les types d'espaces végétaux propres aux franges: des haies buissonnantes et des chemins enherbés le long de cultures maraîchères et céréalières, lieu de prédilection de la grande bardane (Arctium lappa) et de l'armoise commune (Artemisia vulgaris), un bois prenant l'allure d'une futaie domaniale bien entretenue, mêlant chênes, érables et châtaigniers, une promenade plantée avec prairies de fauche où a été semée de la luzerne cultivée (Medicago sativa), des boisements rudéraux impénétrables où s'entremêlent vieux merisiers (Prunus avium), aubépines (Crataegus monogyna), lierre (Hedera helix) et vigne vierge (Parthenocissus quinquefolia), ou encore des friches buissonnantes, voire arborées, isolées et difficiles d'accès le long de l'autoroute A115. Chaque sous-ensemble présente ces spécificités floristiques, à des échelles parfois très fines (tel le remblai enfriché visible sur la photographie ci-dessous). Le fond du paysage est bien dominé par 
l'activité agricole mais il prend l'allure d'une mosaïque d'espaces végétalisés divers et variés.

Figure 5. Vue sur la plaine de Boissy (direction nord-est)

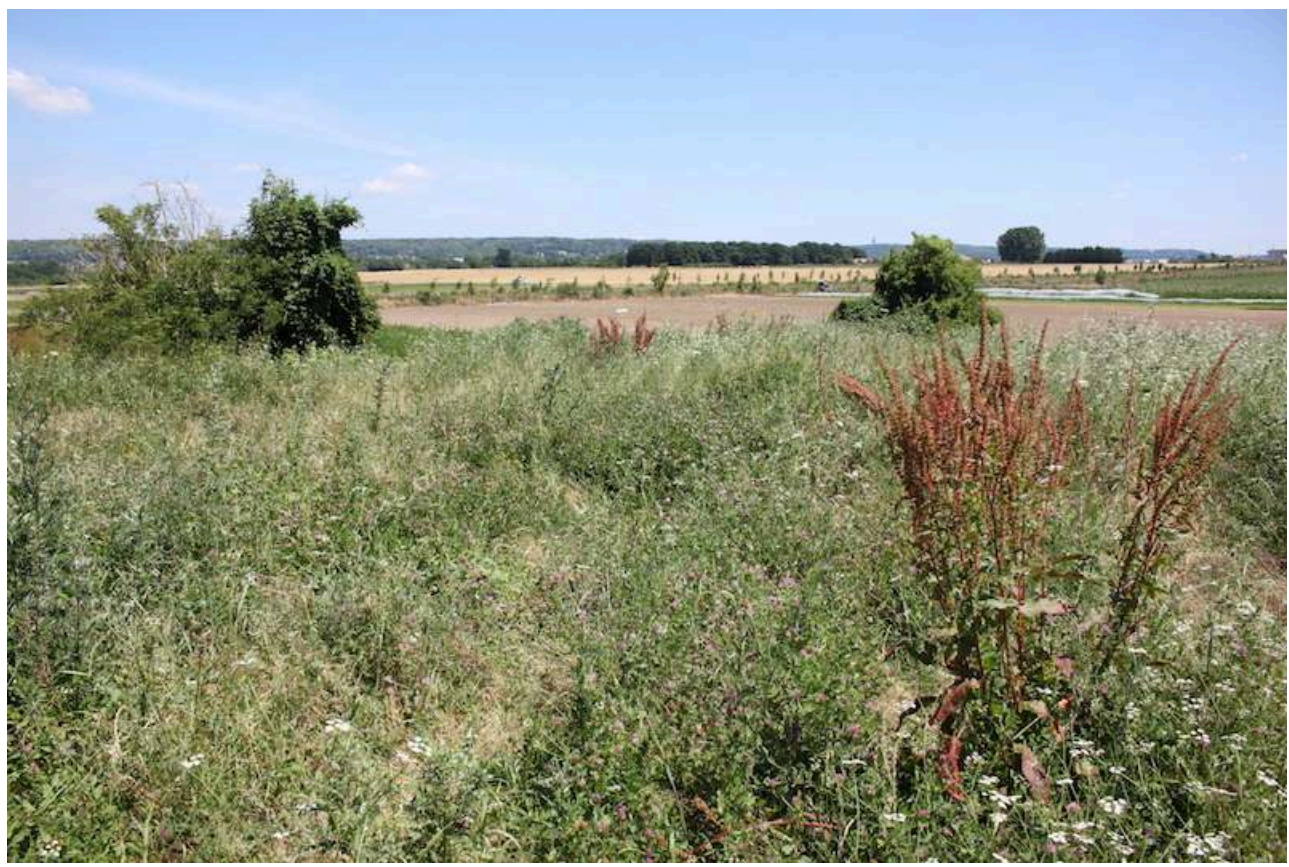

Source : Fabien Roussel, juin 2014.

Un PRIF porté par l'AEV cherche à donner de la cohérence à une grande partie de la zone et permet de maintenir celle-ci hors du développement urbain. Sont réunis ici la plupart des modes de mise en valeur et des usages propres aux espaces végétaux de la ceinture verte: usage récréatif, économique (ressource agricole), patrimonial, paysager... L'AEV entend cependant faire de cette hétérogénéité une synthèse de la campagne aux portes de la ville, s'adressant à une population urbaine. Le panneau qui accueille les promeneurs à l'entrée de la zone classée PRIF indique : « Un petit coin de campagne au milieu des villes ». Le logo de la ville du Plessis-Bouchard va plus loin : alors que c'est bien la « campagne » qui est encerclée dans les faits, il maintient quant à lui l'illusion d'un village dans la campagne.

À contretemps des images véhiculées, la réalité métropolitaine n'est pas loin: la communauté d'agglomération Val-et-Forêt a en projet une zone d'aménagement concertée (ZAC) pour le Nord-Est de la plaine, hors du PRIF, qui doit accueillir nouveaux bureaux et logements. L'espace ne se réclame à aucun moment de la ceinture verte à laquelle il appartient pourtant. La discrétion de celle-ci laisse ici les seuls PRIF comme porteurs d'un projet de mise en valeur des espaces végétaux et agricoles des franges urbaines (Agence des espaces verts de la région Île-de-France 2014). Les PRIF sont situés dans une grande majorité en ceinture verte mais ils y sont cependant éparpillés et de taille très variable. Leurs effets sont donc ponctuels et non globaux. 


\section{Conclusion}

Le promeneur qui parcourrait les quelques $250 \mathrm{~km} \mathrm{du} \mathrm{GR} \mathrm{de} \mathrm{Pays} \mathrm{de} \mathrm{la} \mathrm{Ceinture} \mathrm{verte}$ proposés par la Fédération française de randonnée pédestre au début des années $2000^{5}$ serait bien en peine de voir une quelconque cohérence spatiale et paysagère aux espaces qu'il traverse. Sans doute perplexe en traversant Marne-la-Vallée, CergyPontoise ou Saint-Quentin-en-Yvelines, il n'aurait pas davantage de certitudes en se focalisant sur la couleur verte dans les espaces non bâtis: couleur des chemins agricoles herbacés de la plaine de France, le vert est aussi la couleur des futaies de châtaigniers de la forêt de Montmorency, des promenades arborées des bords de l'Oise, des bois abandonnés de la plaine de Pierrelaye, des friches buissonnantes des bords des autoroutes... L'étude du secteur de Pierrelaye donne un aperçu de l'organisation spatiale de la végétation en ceinture verte : celle-ci est un agrégat spatial, biologique et paysager hétérogène et discontinu d'espaces végétaux aux structures et aux dynamiques plus ou moins naturelles, qui alternent selon l'emprise des matrices paysagères et selon les usages et les fonctions, produisant des paysages diversement valorisés ou valorisables. L'échelle locale est privilégiée pour mener des projets de mise en valeur paysagère de la végétation (comme le montre la réussite des PRIF). Poursuivre l'analyse biogéographique de la végétation dans les marges de l'agglomération est une voie pour relancer une approche globale, à l'échelle régionale, des paysages des franges urbaines et ainsi apporter des réponses à l'ambition inaboutie de la ceinture verte dans un contexte de ville durable relancé.

\section{BIBLIOGRAPHIE}

Agence des espaces verts de la Région Île-de-France, Atlas des périmètres régionaux d'intervention foncière. Édition 2015, URL : http://www.aev-iledefrance.fr/les-missions/amenager-le-territoire/ les-perimetres-regionaux-d-intervention-fonciere.

Alexandre, F., « The Role of Vegetation in the Urban Policies of European Cities in the Age of the Sustainable City ", European Spatial Research and Policy, vol. 20, n 2, 2013, p. 11-27.

Alexandre, F. et Génin, A., "Ceintures vertes autour des grandes métropoles européennes », Formes et Échelles des compositions urbaines, actes des congrès nationaux des sociétés historiques et scientifiques, Paris, édition électronique du Comité des travaux historiques et scientifiques, 2014, p. $75-87$.

Alexandre, F. et Génin, A., Géographie de la végétation terrestre. Modèles hérités, perspectives, concepts et méthodes, Paris, Armand Colin, 2011.

Alexandre, F. et Génin, A., Continu et discontinu dans l'espace géographique, Tours, Presse universitaires François Rabelais, Coll. «Perspectives Villes et Territoires », 2008.

Amati, M., Urban green belts in the twenty-first century, Farnham, Ashgate, Coll. « Urban Planning and Environment », 2008. 
Banzo, M., «L'espace ouvert pour recomposer avec la matérialité de l'espace urbain », Articulo Journal of Urban Research, $\mathrm{n}^{\circ}$ 6, 2015.

Barbieri, N., « La Ceinture verte d'île-de-France, quelle réalité ? , Note rapide sur le bilan du SDRIF, $\mathrm{n}^{\circ}$ 10, Institut d'aménagement et d'urbanisme de la Région Île-de-France, 2002.

Bourget, E., et Le Du-Blayo, L., « Cartographie des paysages : apport à l'analyse des trames vertes et bleues. L'exemple du Pays de Saint-Brieuc », Projets de paysage, ${ }^{\circ}$ 4, juillet 2010, URL : http:// www.projetsdepaysage.fr/fr/

cartographie_des_paysages_apport_a_l_analyse_des_trames_vertes_et_bleues.

Brédif, H. et Pupin, V., « Réévaluer la place de l'agriculture à l'heure du Grand Paris », Annales de géographie, vol. 1, n 683, 2012, p. 43-65.

Clergeau, P. et Blanc, N. (dir.), Trames vertes urbaines : de la recherche scientifique au projet urbain, Paris, Le Moniteur, 2013.

Da Lage, A. et Métaillé, G. (dir.), Dictionnaire de biogéographie végétale, Paris, CNRS édition, 2005.

Darly, S., « Agriculture et patrimoine identitaire des parcs naturels régionaux en Île-de-France : des situations contrastées ", Pour, vol. 2, n 205-206, 2010, p. 103-109.

Forman, R.T.T. et Godron, M., Landscape ecology, New York, John Wiley \& sons, 1986.

Gant, R. L., Robinson, M., et Fazal, S., « Land-use change in the "edgelands" : Policies and pressures in London's rural-urban fringe », Land Use Policy, vol. 28, n 1, 2011, p. 266-279.

Godron, M., Écologie et Évolution du monde vivant, vol 2, L'Échelle crée le phénomène, Paris, L'Harmattan, 2012.

Godron, M. et Joly, H., Dictionnaire du paysage, Paris, Conseil international de la langue française, 2008.

Grison, J.-B., « Le développement périurbain du Pays de France : des influences urbaines différenciées ", Projets de paysage, nº 8, octobre 2012, URL : https://www.projetsdepaysage.fr/fr/ le_d_veloppement_p_riurbain_du_pays_de_france_des_influences_urbaines_diff_renci_es

IAU Île-de-France, « Unités paysagères de la région Île-de-France. Méthodologie, notice d'utilisation de la base de données atlas », Paris, Institut d'aménagement et d'urbanisme de la Région Île-de-France, 2010.

IAU Île-de-France, "Comment traiter les fronts urbains ?", Les Carnets pratiques, Paris, Institut d'aménagement et d'urbanisme de la région Île-de-France, 2010.

Laruelle, N. et Legenne, C., « La ceinture verte, un espace de vie à inventer ", Les Cahiers de l'IAU Île-de-France, Institut d'aménagement et d'urbanisme de la région Île-de-France, $\mathrm{n}^{\circ} 161$, février 2012, p. 97-100.

Laruelle, N. et Legenne, C., « La ceinture verte d'île-de-France, un espace de vie à réinventer. Éléments pour un nouveau partage de l'espace périurbain dense », Paris, Institut d'aménagement et d'urbanisme de la région Île-de-France, 2005.

Lizet, B, « Du terrain vague à la friche paysagée », Ethnologie française, vol. 40, nº 4, 2010, p. 597-608.

Novarina, G. « Ville diffuse et système du vert », Revue de géographie alpine, vol. 91, nº 4, 2003, p. 9-17.

Poulot, M., « Du vert dans le périurbain », Revue électronique des sciences humaines et sociales, mai 2013 
Poulot, M., « Résurgences paysagères et nouvelles économies agricoles dans le périurbain francilien », Projets de paysage, n 8, juillet 2012, URL : https://www.projetsdepaysage.fr/fr/ r_surgences_paysag_res_et_nouvelles_conomies_agricoles_dans_le_p_riurbain_francilien

Poulot, M., « Les territoires périurbains : "fin de partie" pour la géographie rurale ou nouvelles perspectives ?", Géocarrefour, vol. 83, n 4, 2009, p. 269-278.

\section{NOTES}

1. Institution qui venait de recevoir des pouvoirs renforcés dans le cadre des lois de décentralisation.

2. Voir : http://www.iau-idf.fr/fileadmin/user_upload/SIG/cartes_telecharge/PNR_2011.pdf.

3. Voir : https://inpn.mnhn.fr/informations/glossaire/liste/e.

4. Institut géographique national, TOP 100 Tourisme et découverte $\mathrm{n}^{\circ} 190$ «Paris, Chantilly, Fontainebleau ", juillet 2011.

5. Voir Fédération française de randonnée pédestre, GRP de la Ceinture verte d'île-de-France, coll. « Topo Guides », n 100, 2000. L'ouvrage est aujourd'hui introuvable dans le commerce.

\section{RÉSUMÉS}

Le projet d'une ceinture verte en région Île-de-France est resté, faute de contenu, méconnu des acteurs locaux et de la population en général. Nous souhaitons montrer ici que, s'il existe de nombreux projets d'aménagement « verts » des espaces périurbains à l'échelle locale, la ceinture verte est en réalité un espace peu cohérent. Prétexte à signifier une altérité à la ville dense, supposée grise, la couleur verte invoquée nous invite à saisir la clef de lecture de la végétation qui nous conduit vers les espaces végétaux semi-naturels et vers le paysage. En clarifiant le jeu des échelles, en élaborant une typologie emboîtée des paysages de franges, nous proposons ainsi une méthode et des critères de biogéographie végétale qui lient modes d'occupation du sol, flore et paysage et permettent d'envisager la végétation des franges urbaines ainsi que le projet inabouti de ceinture verte sous un nouveau jour.

Due to insufficient content, the green belt project of the Ile-de-France region has remained largely unknown to local stakeholders and the general public. We would like to demonstrate that in spite of many development projects for green spaces in suburban areas at a local level, the green belt lacks coherence spatially. Signifying an alternative to a dense and supposedly grey urban environment the colour green invites us to study vegetation and subsequently seminatural planted and landscaped spaces. By clearly outlining the different scales and defining an interconnected typology of fringe landscapes we propose a method and criteria based on plant bio-geography establishing the links between modes of land occupation, flora and landscape, thus making it possible to envisage vegetation for urban fringes and the unfinished green belt project in a new light. 
INDEX

Mots-clés : ceinture verte, agglomération parisienne, espaces végétaux, paysage, franges urbaines, cohérence spatiale, biologique et paysagère

Keywords : green belt, Parisian agglomeration, planted spaces, landscape, urban fringes, spatial, biological and landscape coherence

\section{AUTEUR}

\section{FABIEN ROUSSEL}

Fabien Roussel est doctorant en géographie, EA7338 Pléiade, université Paris 13, Sorbonne Paris Cité.

fabien.roussel[at]univ-paris13[dot]fr et fab.roussel[at]laposte[dot]net 